\title{
Collapse and the Varieties of Quantifier Variance
}

\author{
Matti Eklund \\ matti.eklund@filosofi.uu.se
}

[draft version - published version in James Miller ed.), The Language of Ontology, Oxford University Press, 2021]

\section{Introduction}

Quantifier variance - a much discussed thesis in recent discussions of ontology — will be my focus here. The thesis has been characterized in many, not obviously equivalent ways. Eventually, the differences between the characterizations will be a theme. Quantifier variance was introduced into the literature, under that name, by Eli Hirsch in a series of articles from the early 2000s and onwards, collected in his (2011), but the general idea has since Hirsch's important work taken on a life of its own (and Hirsch himself credits Putnam with the basic idea). In his recent (2015) - which will be the focus of some of the present discussion - Jared Warren operates with this characterization:

...there are distinct and inequivalent quantifier meanings such that the different parties in various ontological disputes can be interpreted as using different unrestricted existential quantifiers. ${ }^{1}$

In his seminal (2011) book, Ted Sider characterizes quantifier variance as follows:

...ontological deflationists cannot accept that quantifiers carve at the joints. In my view they should go further and uphold quantifier variance, to use Hirsch's term: the claim that there are multiple candidates to be meant by quantifiers, none of which carve perfectly at the joints, but none of which are exceeded in joint-carving by any other quantifier candidate. ${ }^{2}$

This at least provides a general idea of what quantifier variance is about, even if, as we will see, there are importantly different formulations of the thesis.

Quantifier variance is often held to be significant for broader issues regarding ontology. Here is what Warren says about the matter:

\footnotetext{
${ }^{1}$ Warren (2015), p. 241.

${ }^{2}$ Sider (2011), p. 175.
} 
So, when a compositional nihilist says 'there are no chairs!' and a compositional universalist replies 'there are so chairs!', they need not be really and truly disagreeing, since each might be speaking the truth in their own language with their own quantifier. Quantifier variance has been championed by Hilary Putnam and Eli Hirsch, but it has come under heavy fire from ontological realists in every camp. ${ }^{3}$

Sider takes quantifier variance to entail what he calls "ontological deflationism", characterized as follows:

According to this view, the philosopher's question of whether holes exist is confused, because the extraordinary, philosophical sense of the existence of a hole has not been, and cannot be, clearly specified. The only coherent question is the more mundane one of what exists according to ordinary standards. And this mundane question can be settled by a conceptual analysis of ordinary standards; there is no need to resort to the Quinean methodology. Likewise for the other questions of philosophical ontology—questions about the existence of numbers, propositions, events, past and future objects, tables and chairs, and so on. ${ }^{4}$

Somehow, quantifier variance is supposed to have a deflationary upshot for ontology. It is something to be opposed by "ontological realists". Note that it is not obvious how quantifier variance could have any such upshot. Take Warren's characterization of quantifier variance. It says only that there are different quantifier meanings, whence ontological disputants can find themselves having a merely verbal dispute. But why should this be thought to be in conflict with any kind of "realism"? Is it not a philosophically fairly boring fact that disputants can happen to speak past each other?

What I will do here is to discuss how quantifier variance is best understood, and how to think about its purported broader significance for ontology. I will do this through discussing two arguments that prominently have been used against the thesis of quantifier variance, the collapse argument and the so-called Eklund-Hawthorne argument. ${ }^{5}$ The upshot of the discussion will be that there are different kinds of quantifier variance theses, with different sorts of implications for ontology and not all equally vulnerable to the counterarguments proposed. Thus put, the project of the paper may sound bland. But I believe that distinguishing between the different quantifier variance theses both helps sort out significant confusions in the literature, and helps put the spotlight on importantly different ways in which one might be a ontological deflationist.

\footnotetext{
${ }^{3}$ Warren (2015), p. 241.

${ }^{4}$ Sider (2011), p. 167.

5 I have elsewhere used labels such as "the Tarskian argument" (Eklund 2008) and "the semantic argument" (Eklund 2009) for the latter.
} 
In the background of the discussion of quantifier variance is the ("Quinean") assumption that existence is adequately expressed using so-called existential quantification. I too will take this assumption for granted. ${ }^{6}$ For someone who rejects the assumption, seeing existential quantification as ontologically innocent and seeing existence as better expressed using an existence predicate, things will look different. In her setting, the relevant variance thesis is not quantifier variance but a corresponding thesis about what different existence predicates there are. In occasional footnotes, I will remark on how differently things look from that perspective.

\section{The collapse argument}

Warren's formulation of quantifier variance invites the question what it is for there to be different unrestricted existential quantifiers. Warren addresses this:

... according to quantifier variance, an expression in any given language is an existential quantifier expression just in case it plays the inferential role of the existential quantifier, i.e., if it obeys the standard introduction and elimination rules for the existential quantifier. ${ }^{7}$

Quantifier variance as Warren states it then involves the claim that there are non-coextensive expressions which all play the inferential role standardly associated with the existential quantifier. ${ }^{8}$

This thesis is disproven if it can be shown that any two expressions with this inferential role must be equivalent. This is what the so-called collapse argument seeks to show. The collapse argument goes back to work by Harris (1982), and has been used by, e.g., Hale and Wright (2009) in attempts to refute quantifier variance.

Warren uses the dispute between (mereological) universalists and (mereological) nihilists in metaphysics to illustrate quantifier variance. (Mereological nihilism is the thesis that no matter what some objects are, those objects do not have a sum. Mereological universalism is the thesis that no matter what some objects are, those objects do have a sum.) Quantifier variance, as applied to this dispute, says that there is a language, Universalese, where the universalist's utterances come out true, and a language, Nihilese, where the nihilist's utterances come out true; and these languages employ different existential quantifier expressions, in the sense characterized. While it may be useful to have a concrete example like universalism/nihilism in mind, I will speak instead of "Biglish" and "Smallish", where Biglish corresponds to a more liberal ontological view and Smallish corresponds to a more restrictive one; and I will reformulate examples accordingly. ${ }^{9}$

\footnotetext{
${ }^{6}$ For some discussion of the reasonableness of such an assumption, see Eklund (2014).

${ }^{7}$ Warren (2015), p. 242.

${ }^{8}$ In a non-Quinean setting things would look very different. What would be the inferential role associated with an existence predicate of the kind the non-Quinean uses?

${ }^{9}$ I here follow Sider (2011); see p. 181.
} 
Working with this example, here is the collapse argument as Warren presents it, in broadest outline. I use " $\exists_{\mathrm{B}}$ " for the Biglish quantifier and " $\exists_{\mathrm{S}}$ " for the Smallish quantifier. Suppose

$$
\text { (1) } \exists \text { BX chair(x) }
$$

Then let " $\alpha$ " be a name of an object witnessing the truth of (1). We then have, by the standard elimination rule for the quantifier,

(2) $\operatorname{chair}(\alpha)$

If the Smallish quantifier is indeed an existential quantifier, then, the argument runs, it obeys the standard introduction rule for an existential quantifier. But then from (2) we can infer

$$
\text { (3) } \exists \mathrm{s} x \text { chair(x) }
$$

But then, Warren says, stating the upshot of the argument, "We've established, in [Smallish]... that " $\left[\exists_{\mathrm{s}}\right] \mathrm{x}$ chair(x)" is true. But this is a disaster, since, by stipulation, " $\left[\exists_{\mathrm{s}}\right] \mathrm{x}$ chair(x)" is false in [Smallish]." 10

Warren notes that as presented, the collapse argument is based on a non sequitur: sentences (1) is a sentence only of Biglish and (3) a sentence only of Smallish - so in which language were we supposed to carry out the argument?

There is no doubt that there is a problem. But can the argument be repaired? One obvious strategy for the friend of the collapse argument is to say that if indeed there are such languages as Smallish and Biglish one should be able to combine the two languages, and then in the combined language argue that the two supposedly inequivalent quantifiers are equivalent.

Warren considers simply adding sentences like (1) to Smallish and says about this:

...we could add this sentence to [Smallish], but then we'd also need to add the [Biglish] quantifier rules to complete the collapse argument and this would alter the language and thereby block the reductio of quantifier variance. ${ }^{11}$

This may be somewhat quick. Warren notes that just because the collapse argument goes through in the combined language Smallish $\cup$ Biglish does not immediately mean that there cannot be a language

\footnotetext{
${ }^{10}$ Warren (2015), p. 243. Warren's own exposition is considerably more careful and elaborate. I have tried to distil the argument down to bare essentials.

${ }^{11}$ Warren (2015), p. 246.
} 
Smallish as envisaged. That is surely correct. But the needed extra premise - if there is a language Smallish as envisaged then there must also be a language Smallish $\cup$ Biglish with two different existential quantifiers - is, although far from obviously true, rather natural to accept. If there are suchand-such meanings for expressions to have, why cannot expressions with these different meanings cohabit - exist in the same language? (There may be good reasons not to accept the general principle that in any case where there are expressions with different meanings, they can cohabit. The semantic paradoxes present problems. But in the present case we would be dealing with paradox-independent restrictions on the general principle.)

Whatever in the end to say about Warren's objection, Warren's own preferred suggestion is that a sentence like (1) of Biglish be translated non-homophonically into Smallish, using "the plural quantifiers and predicates of [Smallish] to stand proxy for the singular quantifiers and predicates of [Biglish]". ${ }^{12}$ The reason this is supposed to help is that if we replace the sentence of Biglish in the above argument with its non-homophonic translation into Smallish, then the reasoning of the argument does not go through.

When assessing this suggestion, let us distinguish between two theses the friend of quantifier variance might maintain regarding what languages there are. The weak thesis is that there are languages like Biglish and Smallish as envisaged, in the sense that there are two languages like ordinary language except that the "biggist's" (e.g. the universalist's) characteristic utterances are true in the one but the smallist's (e.g. the nihilist's) characteristic utterances are true in the other. The strong thesis says that there can be such languages, while both languages employ what can be regarded as, in some sense, different unrestricted existential first-order quantifiers, expressions whose meanings are unrestricted existential quantifier meanings. ${ }^{13}$

The difference between the thesis is that the weak thesis does not say anything about what kinds of meanings the relevant expressions have. The friend of the weak thesis will have to say something also of utterances of sentences that on the surface involve unrestricted existential quantification, but there is nothing in the thesis that commits her in any way regarding what kinds of meanings these sentences and the expressions they contain actually have.

It can of course be questioned what the strong thesis even means. What does it even meant to say that there are these different meanings? But whatever exactly the strong thesis amounts to, the supposed difference between the two theses should be plain. The weak thesis in no way commits to there being different unrestricted existential first-order quantifiers. The weak thesis could be true for example because one of the parties does not use "there are" to express a quantifier meaning. The friend of the weak thesis can even completely dismiss the question of which expressions have quantifier meanings; for the friend of the strong thesis, by contrast, that issue is central.

\footnotetext{
${ }^{12}$ Warren (2015), p. 246f.

${ }^{13}$ In the non-Quinean setting, the strong thesis would be the claim - however it is to be cashed out - that different languages can contain co-extensive predicates which all count as existence predicates.
} 
Warren's translation suggestion illustrates the distinction between the weak and strong theses. Assuming the plural translation suggested by Warren to be otherwise workable, the weak thesis may well be vindicated. But the plural translation is not helpful when it comes to vindicating the strong thesis. If we speak Smallish, then we understand the speaker of Biglish not as using a different unrestricted existential first-order quantifier but as employing different linguistic means.

Recall Warren's own statement of quantifier variance: “Quantifier variance is a metaontological view according to which there are distinct and inequivalent quantifier meanings such that the different parties in various ontological disputes can be interpreted as using different unrestricted existential quantifiers." Taken at face value, the formulation suggests that Warren is concerned with the strong thesis: quantifier variance as he presents it requires that the parties use different unrestricted existential quantifiers. The mere fact that the disputants speak different languages, and mean different things by locutions like "there are", ${ }^{14}$ does not mean that they use different unrestricted quantifiers: for it may be that at least one of the disputants does not use an unrestricted quantifier at all. And to stress, if Warren is concerned with the strong thesis, what he says about translation is problematic. What language is the quantifier variantist herself supposed to speak? If she speaks Smallish, then given Warren's suggestion about translation, she can't say of speakers of Biglish that they "use a different unrestricted existential quantifier". (If she speaks Biglish, a different concern arises: must she not interpret the speaker of Smallish as using a restricted existential quantifier.)

Warren's explicit formulation of quantifier variance suggests that he is concerned with the strong thesis, but his own reasoning reveals that he is concerned with the weak thesis.

\section{Free logic}

In a different context, Jason Turner suggests responding to the collapse argument by adopting the quantifier rules of a version of free logic instead of classical logic. Turner's proposal is that, using the appropriate logic, one cannot infer something of the form " $\exists \mathrm{xF}(\mathrm{x})$ " simply from " $\mathrm{F}(\mathrm{t})$ "; one also needs " $\exists \mathrm{x}(\mathrm{x}=\mathrm{t})$ ". ${ }^{15}$ This blocks the collapse argument: we cannot infer

(3) $\exists$ s $x$ chair(x)

from

(2) $\operatorname{chair}(\alpha)$,

\footnotetext{
${ }^{14}$ I will throughout follow established custom in philosophical discussions of ontology and treat "there are" as itself a quantifier expression. This isn't true to the linguistic facts, but it doesn't matter. The important thing is just to speak of some natural language expression which is a quantifier, and "there are" is the standard example used.

${ }^{15}$ Turner (2010), p $25 f$.
} 
unless we have

$$
\exists_{\mathrm{S} x}(\mathrm{x}=\alpha)
$$

In Turner's context, this seems clearly to be the right response. He is concerned to defend a version of what has come to be called ontological pluralism, the thesis that there are different modes of being. As Turner and Kris McDaniel develop the thesis, it amounts to claiming that there are different natural quantifiers: for each mode of being, there is a natural quantifier ranging over exactly what enjoys that mode of being. ${ }^{16}$ Then one can reason that even if something is in the range over the quantifier ranging over (say) all abstracta, it is not in the range of a quantifier ranging over all concreta - and adopting free logic the way Turner does reflects this.

The quantifier variance setting is different. In the case of quantifier variance, the hypothesis is that there are - at least in some sense, recall the weak/strong distinction - different unrestricted quantifiers. This does not mean that Turner's strategy cannot be used. I will return to it. What it does mean is that Turner's strategy is more problematic in that case.

\section{Quantifier variance theses}

It is worth comparing some different characterizations of quantifier variance found in literature in light of the distinction between the weak and the strong theses. In the next section I will turn to the question of what sort of quantifier variance thesis is more philosophically significant.

Hirsch's own formulations consistently suggest the weak thesis. In the introduction to his (2011), he states, "Quantifier variantism says that there is no uniquely best ontological language with which to describe the world. I take this to imply that (i) there are a number of possible truthconditionally equivalent ontological languages, and (ii) these languages are of equal metaphysical merit." ${ }^{\prime 17}$ In his (2002), one of his earliest papers defending quantifier variance, he characterizes quantifier variance by saying,

Putnam's doctrine of quantifier variance implies that the expression "there exists something" can be interpreted in a way that makes the sentence true or in a way that makes the sentence false.

Since both interpretations are available to us, we have a choice between operating with a concept of "the existence of something" that satisfies the mereologist or operating with a different concept that satisfies the anti-mereologist. ${ }^{18}$

\footnotetext{
${ }^{16}$ See, e.g., McDaniel (2017) and Turner (2010, 2012).

${ }^{17}$ Hirsch (2011), p. xiv.

${ }^{18}$ Hirsch (2002), p. 69.
} 
In a (2009) paper, the characterization is that quantifier variance demands that it is possible "that quantifier-like expressions in different languages may have different semantic functions; they may contribute differently to the characters of sentences". ${ }^{19}$ Nowhere do we see the characteristic demand of the strong thesis: that the quantifier-like expressions of the different expressions really have quantifier meanings.

Here is the formulation of quantifier variance that Warren and Hirsch operate with in their joint (2019):

A modest form of quantifier variance says that, in many ontological disputes, if you are on one side of the dispute, you can conceive of a possible language whose sentences are true just in case philosophers on the other side of the dispute would be willing to assert them. More explicitly, this means that, if your opponents hold the $\mathrm{X}$-position you can conceive of a possible $\mathrm{X}$-language such that ". . . in any context of utterance, [a] speaker of the $\mathrm{X}$-language can both reasonably and truthfully assert the same (phonetically individuated) sentences (with respect to a possible world) that the X-philosophers assert... ${ }^{20}$

I am actually not sure what Hirsch and Warren have in mind when they call this form of quantifier variance "modest", but their statement does correspond to what I earlier called the weak thesis.

Ted Sider (2007) characterizes "Neo-Carnapian quantifier variance" as follows:

There is a class, $\mathrm{C}$, containing equally and maximally natural candidate meanings for quantifier expressions, in that: (i) no member of $\mathrm{C}$ is more natural than any other member of $\mathrm{C}$, and (ii) no candidate meaning for quantifier expressions that is not in $\mathrm{C}$ is as natural as any member of $\mathrm{C}$. Each position in the debate over the ontology of composite material objects comes out true under some member of $\mathrm{C}^{21}$

Sider here crucially speaks of "meanings for quantifier expressions", and seamlessly goes on to discss these as "quantifier meanings". For quantifier variance as Sider conceives of it to be true, there must be different kinds of quantifier meanings as described - and Sider (2007) is then largely devoted to a discussion of what this might mean. In that work, Sider then focuses on the strong thesis.

In his later (2011) book, however, Sider explicitly distances himself from the strong thesis. The official characterization of quantifier variance, quoted above, is that it is "the claim that there are multiple candidates to be meant by quantifiers, none of which carve perfectly at the joints, but none of which are exceeded in joint-carving by any other quantifier candidate". This does not clearly indicate

\footnotetext{
${ }^{19}$ Hirsch (2009), p. 239.

${ }^{20}$ Hirsch and Warren (2019), p. 592.

${ }^{21}$ Sider (2007), p. 209.
} 
whether it is the strong or the weak thesis Sider has in mind. But Sider goes on to reflect that what friends of quantifier variance "really care about is vindicated simply by the claims that: i) there are multiple possible languages of this sort; ii) quantificational claims can have different truth-values in the different languages; and iii) none of the languages are more joint-carving than the rest" ${ }^{22}$ Hence, it need not bother quantifier variantists - in Sider's (2011) sense - if what is superficially a quantifier expression in another language gets interpreted in such a way as not to have a quantifier meaning.

My remarks here have focused on relating the different formulations to the weak and strong theses characterized. But there are obviously other salient differences as well. Some characterizations - the one from the introduction to Hirsch's (2011) and Sider's two characterizations - explicitly mention something about being best, or most natural or most joint-carving. Other characterizations Hirsch's other characterizations and the one from Hirsch and Warren's joint paper - do not mention anything of the kind.

\section{Philosophical significance}

Is the weak thesis or the strong thesis more philosophically significant? Or, better, since the strong thesis entails the weak one: what, if anything, of philosophical significance does the strong thesis add to what already the weak thesis provides?

The answer may depend on ulterior philosophical motives. Let me in what follows describe some different outlooks on ontology, all in some way related to the broad theme of quantifier variance, and how they relate to the weak and strong theses.

Suppose that philosopher $\mathrm{X}$ sets out to show that some ontological disputes are verbal, in the sense that the propositions the disputants affirm actually are consistent with each other, contrary to appearances. Let me call the thesis that $\mathrm{X}$ defends verbalism. On her view, some or many ontologists speak past each other, speaking somewhat different languages and not noticing that they do so. Notice that this verbalism is compatible with taking ontology very seriously. Consider a view like Ted Sider's ontological realism, according to which there is a privileged quantifier and the proper way to do ontology is to consider what exists in the sense of the privileged quantifier. An ontological realist can take in her stride the point that ontologists tend to speak past each other. That descriptive point about how ontology tends to be practiced is consistent with the significance and feasibility of her envisaged project of investigating what exists in the sense of the privileged quantifier. (It would be different if it were plainly impossible to have an ontological dispute which is non-verbal: but such a strong verbalist claim - much stronger than a claim to the effect that ontological disputes tend to be verbal - falls on its own implausibility. ${ }^{23}$ )

\footnotetext{
${ }^{22}$ Sider (2011), p. 176.

${ }^{23}$ On this issue regarding verbalism, see further my (2016).
} 
For X's purposes, it does not immediately matter whether it can be said that these philosophers use different unrestricted existential quantifiers. All that matters is that they use "there are" and similar expressions with different meanings. Perhaps not more than one of these meanings is a meaning of an unrestricted quantifier - but why should that be a concern? Only the weak thesis is immediately relevant. $\mathrm{X}$ is in no way committed to the strong thesis. What she says is compatible with the strong thesis, and there could be independent reasons to believe the strong thesis, but that is another matter.

Compare now philosopher Y, seeking to defend a more principled deflationary view. On Y's view, there is no metaphysically privileged quantifier meaning, but there simply are different languages, with different quantifiers, all equally apt for the task of describing the world. No language, and no quantifier, is metaphysically privileged over all others. Y's view deflates ontology in the following sense. While ontology might have seemed deep when it was thought that questions about what exists had some special metaphysical standing, one can, given Y's view, see that they don't (a language using "exists" could without loss be replaced by one of the alternative languages), and no other notion of existence has that special metaphysical standing either. Notice that Y's view has nothing to do with verbalism. It is perfectly consistent with Y's view that disputing ontologists seldom or never are talking past each other but instead typically use expressions with the same meanings.

I remarked above on the fact that some but not all of the characterizations of quantifier variance include a clause about a best, or most natural, or most joint-carving quantifier meaning. Any such clause would be beside the point for X's purposes; but such clauses go straight to the heart of what $\mathrm{Y}$ is after.

Recall too Sider's saying that according to ontological deflationism, "the extraordinary, philosophical sense" of "exists" simply "cannot be" clearly specified, and the only coherent question in the vicinity "is the more mundane one of what exists according to ordinary standards". Ontological deflationism in this sense can be vindicated by Y's view. Y is naturally understood as saying that there are different mundane existence concepts but no "extraordinary" one. X's view, by contrast, is of no help in vindicating it, since X's view doesn't immediately do anything to cast doubt on there being a special philosophical sense of "exists".

When it comes to Y's view it is clearly the strong thesis which is relevant, for Y's view, as stated, is centrally concerned with what quantifier meanings there are. Moreover, Y's thesis seems more philosophically significant than X's, since Y's thesis is of greater principled significance for ontology.

Recall here Turner's appeal to free logic. How to evaluate this appeal depends crucially on whether it is X's or Y's view that is at issue. If it is X's verbalism that is at issue, then the question is whether the disputants's respective quantifiers are governed by the rules of free logic - or better, whether when the disputations state in their respective languages what the rules are, they say things that sound free logic-y. If it is Y's thesis that is at issue, the question is what sorts of rules the "best" quantifiers are governed by, classical rules or free logic rules. 
The theoretical map can be made more complicated. There is a view in the ballpark of Y's but relevantly different that deserves bringing up. Why focus on Y's view as stated - which centrally concerns what quantifier meanings there are - instead of a view in the same ballpark, which rather says,

A language which employs our existence concept is just one among many possible languages there are, languages that instead use other kinds of representational means describe the world equally well, and no kind of possible language is metaphysically privileged over all others.

Call this Z's view. Z's view is of the same general kind as Y's. Z, like Y, presents a thesis about what possible languages there are and how they relate; contrast $\mathrm{X}$, who makes a claim about how actual debates are prosecuted. The difference with Y's view is that Z's view does not commit itself to the other languages employing expressions with quantifier meanings. The other languages need not employ anything like a counterpart of the quantifier we employ.

Note that there are importantly different kinds of views of Z's kind. On one type of view of that kind, there are possible languages as good as ours that employ nothing like ordinary quantification. On another type of view of Z's kind, it is only said that there are possible languages as good as ours where the counterpart of the existential quantifier is only somewhat unlike the ordinary existential quantifier - maybe it only obeys slightly different inference rules. A reason for emphasizing the possibility of the latter kind of view is that already slight differences in what inferences rules are obeyed affects the applicability of the collapse argument.

Z's view appears to pack the same deflationary punch as Y's view: it deflates ontology in the same way. What is so special about questions about what exists, if we can equally well state our theory of the world in, so to speak, existence-free terms? ${ }^{24}$ But Z's view, like X's, needs only the weak thesis - despite the other differences there are between $\mathrm{X}$ and $\mathrm{Z}$.

Could Y's view possibly have a significance which Z's view lacks? Perhaps like this. Z's view alone does not preclude that there is only one way - or one best way - to describe the world in quantificational terms. One can then say, even given Z's view, that if the world is to be described in quantificational terms at all, then it is a given how that is to be done. Y's view does preclude this. To the extent that this is an important difference, Y's view does have significance which Z's view lacks.

Whatever in the end to say about the differences between the views of $\mathrm{Y}$ and $\mathrm{Z}$, there is a significant distinction between the view of $\mathrm{X}$ on the one hand and the views of $\mathrm{Y}$ and $\mathrm{Z}$ on the other.

\footnotetext{
${ }^{24}$ If, in order to express existence, a quantifier must be governed by some definite set of rules, then a position like Z's should seem still more reasonable. Suppose it is held, for whatever reason, that the inference rules a quantifier must obey to express existence are one familiar from classical loic. Then what of a language whose quantifier obey instead, e.g., the rules that Turner discusses? Such a quantifier does not express existence, given the assumption. But it is very much a further question whether this means that the language is thereby describes the world less well.
} 
X's claim concerns what languages are actually spoken by some theorists; Y's and Z's claims concern possible languages and the relation between them. X's claim in no way involves judgments about which languages are in some sense better than others; Y's and Z's claims are centrally concerned with such judgments. While all three envisaged theorists focus on language and approach questions of ontology via language, they do so in fundamentally different ways.

\section{Dorr's template}

I have used the views of philosophers $\mathrm{X}-\mathrm{Z}$ to illustrate the difference between the weak and the strong theses, and the relationship between these theses and what significance different theses in the vicinity of "quantifier variance" can be said to have. The collapse argument itself has temporarily disappeared from view; let me now return to that argument.

Cian Dorr (2014) presents the following general strategy behind using a collapse argument against quantifier variance.

Interpretation premise: ' $\exists$ ' has the “ $\exists$-Intro" and " $\exists$-Elim” properties in the languages of each of $\mathrm{L}_{1}, \ldots, \mathrm{L}_{\mathrm{n}}$.

Theorem: If $\exists_{1}$ has these properties in $\mathrm{L}_{1}$ and $\exists_{2}$ has them in $\mathrm{L}_{2}$, and (...possible further conditions on $L_{1}$ and $L_{2} \ldots$ ), then $\exists_{1}$ in $L_{1}$ is "equivalent" to $\exists_{2}$ in $L_{2}$.

Conclusion: The meanings of ' $\exists$ ' in the languages of $\mathrm{L}_{1}, \ldots, \mathrm{L}_{\mathrm{n}}$ are all "equivalent" ( . . provided that these languages pairwise satisfy the relevant further conditions). ${ }^{25}$

There are, as Dorr emphasizes, importantly different formulations of the " $\exists$-Intro" and " $\exists$-Elim" rules, and one gets different versions of the argument depending on what rules one focuses on (and perhaps different versions of quantifier variance depending on what rules one takes as characterizing an existential quantifier). Each version must be evaluated separately.

Using Dorr's template, let me revisit the weak and strong theses. Dorr's template involves certain place-holders, like the talk of satisfying "relevant further conditions". But even without getting into what the further conditions might be, we can make some remarks. Given the strong thesis, we have a natural motivation for the interpretation premise. It is natural to think that for two expressions to express unrestricted existential quantifier meanings is for them both to satisfy such-and-such introduction and elimination rules. Given the weak thesis, there is not the same immediate reason to suppose that the expressions in question satisfy the same inference rules. Insofar as there is reason to

\footnotetext{
${ }^{25}$ Adapted from Dorr (2014), p. 508f. Dorr presents the strategy in the case of a simpler example, disjunction. I have reformulated in terms of existential quantification.
} 
accept the interpretation premise, it comes from a different source: direct attention to how the specific languages are actually used.

One complication for the friend of the collapse argument is that it is not sufficient for her purposes that in the different languages same-sounding statements of the rules come out true: for such a statement might include an expression, like "singular term", which itself varies in meaning across languages. Expressions that fall under "singular term" of one language might fail to fall under "singular term" of another. The speaker of Biglish will talk of her "singular terms referring to tables", but a speaker of Smallish employing Warren's plural translation will describe these same terms not as "singular terms" but as "plural terms".

To illustrate, consider an argument that Michaela McSweeney (2017) offers regarding quantifier variance. McSweeney's overall aim is to provide a criterion for when we are justified in holding two theories to be metaphysically equivalent. The details of McSweeney's positive proposal do not matter for present purposes, but briefly, her proposal centers on there being a common definitional extension of these theories. ${ }^{26}$

Regarding quantifier variance, she says,

The quantifiers occupy the exact same proof-theoretic role for the universalist and the nihilist. Both the nihilist and the universalist will themselves say that they are using our regular old semantics and inference rules for the quantifiers. And if they are right that they are doing so, then there is a very quick argument that there can be no [common definitional extension] of universalism and nihilism. Let's call universalism $T_{u}$ and nihilism $T_{n}$. The quantifiers, exists ${ }_{u}$ in $L_{u}$ and exists ${ }_{n}$ in $L_{n}$, have the same inference rules. When we move to $\mathrm{L}+$, we cannot change these inference rules, for [that would violate] the requirement that $\mathrm{L}+$ be a superset of $\mathrm{L}_{\mathrm{u}}$ (and of $\mathrm{L}_{\mathrm{n}}$ ). ${ }^{27}$

And then, McSweeney concludes, exists ${ }_{\mathrm{u}}$-sentences and exists ${ }_{\mathrm{n}}$-sentences will be interderivable in $\mathrm{L}+$, which contradicts the quantifier variantist claim. But consider the crucial claim that "the nihilist and the universalist will themselves say that they are using our regular old semantics and inference rules for the quantifiers". A quantifier variantist could in principle respond that more care is needed: while these theorists will utter same-sounding sentences of their respective languages when stating how their quantifiers work, we cannot conclude from this that the rules are the same, for some expressions employed in these statements may well differ in meaning.

Above I distinguished between the views of philosophers X, Y and Z. Perhaps unsurprisingly, this distinction does not always map neatly onto the views of actual philosophers, even though I think the views of $\mathrm{X}, \mathrm{Y}$ and $\mathrm{Z}$ are the natural views in the vicinity. Consider for example Dorr (2014) and

\footnotetext{
${ }^{26}$ To be a bit more precise, McSweeney uses a tweaked notion of common definitional extension (see 2017, p. 279f), but these details do not matter for present purposes.

${ }^{27}$ McSweeney (2017), p. 282f.
} 
John Hawthorne (2006). These discussions both target the same sort of view, which, following Dorr, I will call "conciliationism". Dorr and Hawthorne characterize certain idealized communities, such that these communities use certain expressions and sentences differently from each other, and the conciliationist view is that all the communities express truths using these sentences. Neither Dorr nor Hawthorne demand that the communities employ different quantifier meanings (as opposed to using merely using expressions like "something" or "there are" to mean different things). Dorr's article is an extended discussion of the collapse argument. He discusses, roughly, under what conditions it is plausible that these communities mean such things by their quantifier expressions that a version of the collapse argument can be employed to show that their quantifier expressions are coextensive. He focuses on different statements of the rules of inference governing the quantifier expressions, and on the conceptual resources used in characterizing them. Hawthorne's discussion touches upon many different things, but among other things, he presents a version of the argument against quantifier variance to be presented in the next section.

Neither Dorr nor Hawthorne is concerned with the question that theorists $\mathrm{Y}$ and $\mathrm{Z}$ are concerned with, about what kind of language, if any, is in a certain sense best. Dorr even explicitly disavows talk of metaphysical merit, on the ground that he does not see a distinction between metaphysical and pragmatic grounds for preferring a language. The best understanding is that they instead are concerned with what $\mathrm{X}$ is concerned with. But their discussions are conducted as regarding certain idealized communities, so it is not entirely straightforward to apply the supposed lessons of their discussions to actual disputes, and thus to establish the sort of verbalist thesis that $\mathrm{X}$ is interested in.

It may be suggested that their aim is different from those of $\mathrm{X}-\mathrm{Z}$ but what could the alternative aim reasonably be? They are not making claims about actual theorists and their languages, and they are not evaluating the merits of the merely possible languages they discuss.

\section{The Eklund-Hawthorne argument}

Thus far I have focused on the so-called collapse argument, which seeks to use the basic argumentative technique of Harris (1982) to show that two quantifiers obeying the standard inference rules are equivalent, in a way that disproves the doctrine of quantifier variance. I will now turn to another argument which has been used against quantifier variance, the so-called Eklund-Hawthorne argument. I will show that the very same issues arise with respect to this argument.

Here is the Eklund-Hawthorne argument as set out by Sider:

Consider two characters, Big and Small. Big speaks an "expansive" language, Biglish, in which speakers freely quantify over tables. Big introduces a name, 'a,' for a table, and thus accepts 'Table(a).' Small speaks a "smaller' language, Smallish, in which speakers refuse to quantify over tables. But Small is a quantifier variantist, and thinks that he does not genuinely disagree with Big. 
So Small says to himself, speaking in Smallish: "Even though there are no tables, the sentence 'Table(a)' is true in Biglish." But this commits Small — and all quantifier variantists, who must accept the scenario as described - to rejecting familiar Tarskian ideas about semantics... In order for " "Table(a)" is true in Biglish' to be true in Smallish, "There is something that "a" denotes in Biglish' must be true in Smallish. But what would this object—in the Smallish sense of "object"be, if not a table? ${ }^{28}$

I prefer to use Sider's formulation to work with over, for example, one of my own for a number of reasons. It is a nice formulation. Those who discuss the argument frequently turn to this formulation. And if I worked with one of my own formulations, I would have a harder time resisting a temptation to get bogged down in, and try to defend, details regarding the formulation. ${ }^{29}$

The argument purports to present a certain problem for the quantifier variantist. But exactly what is the problem?

In a joint paper critically discussing the Eklund-Hawthorne argument, Hirsch and Warren (2019) present the argument as follows (they speak of universalism and nihilism - as elsewhere, I have replaced such talk by generic talk of "big" and "small"):

1. We are in the position to acknowledge the possibility of there being a language Biglish corresponding to the standpoint of the Biggists.

2. If it is impossible in one language to formulate a Tarskian semantics for another language, then speakers of the first language are in no position to acknowledge the possibility of the second language.

3. It is impossible to formulate in Smallish a Tarskian semantics for Biglish.

4. Therefore, our language cannot be Smallish.

5. Therefore, Smallism is not true. ${ }^{30}$

As stated in Hirsch and Warren's numbered argument, the conclusion is just that nihilism is not true. And the mere fact that nihilism is untrue is compatible with quantifier variance. Quantifier variance, however exactly it is to be characterized, just says that there are different languages where different ontological sentences come out true. This is perfectly compatible with there being a fact of the matter regarding what sentences come out true in our language and hence which theses are true. But they go on to reflect, separately,

\footnotetext{
${ }^{28}$ Sider (2011), p. 181. My own presentations of the argument are found in e.g. (2008) and (2009), and Hawthorne's presentation is in his (2006).

${ }^{29}$ Turner's move is as relevant here as in the case of the original collapse argument. In a free logic setting, I cannot even reason from the truth of an atomic sentence in my own language to the conclusion that a singular term in the sentence refers to something.

${ }^{30}$ Hirsch and Warren (2019), p. 593.
} 
If the argument succeeds it will generalize to show that our own language must be, in a sense, as "big" as any language that we can make intelligible to ourselves....[This] certainly means that we cannot view another possible language' s ontology as being "bigger" than our own, as modest variantism often requires. ${ }^{31}$

We can state this as a new conclusion:

6. Therefore, we cannot view another possible language's ontology as being "bigger" than our own, as modest variantism often requires.

They turn to challenge the argument by challenging premise 3, and they do so by formulating a "Tarskian semantics", where a semantics is "Tarskian" in the sense at issue "just in case it generates truth conditions for sentences compositionally in the familiar fashion by mapping items from each syntactic category to objects of the appropriate type - names map (singularly) to objects, monadic predicates map (dividedly) to the objects they are true of, and so on and so forth". ${ }^{32}$

The specific way that they construct a Tarskian semantics involves finding entities quantified over in $\mathrm{N}$ such that the singular terms of $\mathrm{U}$ occurring in true atomic sentences refer to such entities. But to rely on this strategy is to overlook a central element of the Eklund-Hawthorne argument. Consider again the bit that comes after conclusion 5. To repeat: Hirsch and Warren agree that the upshot of the argument is "that we cannot view another possible language's ontology as being "bigger" than our own, as modest variantism often requires". But if one makes sense of the supposedly bigger language $U$ by interpreting its referring terms as referring to entities quantified over in one's own language, one makes sense of it, but only at the expense of treating it as not in fact bigger.

This immediately raises a further question: in what sense can one regard "another possible language's ontology as being "bigger"” to begin with? The most straightforward sense in which another language's ontology can be bigger is if there are things that are in the range of that language's quantifiers that aren't in the range of the quantifiers in one's own language. But thus understood, the claim that another language's ontology is bigger is obviously self-refuting: one quantifies over the entities supposedly only quantified over in the other languages. Hirsch and Warren might then say that the only reasonable sense that can be made of the claim is something like: one language is in the relevant sense "bigger" than another if what superficially are quantifiers and singular terms in both are such that superficially the domain of quantification of the former contains more than the domain of quantification of the latter, regardless of what deeper semantic differences there are beneath the

\footnotetext{
${ }^{31}$ Hirsch and Warren (2019), p. 593.

${ }^{32}$ Hirsch and Warren (2019), p. 594.
} 
surface appearances. A different view, however, would be that one need not and should not focus merely on superficial features, but one can focus on deeper similarities regarding the meanings of the expressions, for example similarities in what rules they obey.

Having rejected premise 3 in their original formulation of the Eklund-Hawthorne argument, Hirsch and Warren go on to consider the possibility that the demand is not just for some Tarskian semantics or other, but for a Tarskian semantics that satisfies some further condition.

One possible demand is that a semantics for a language should provide synonyms for the expressions of the object language. Hirsch and Warren reject this demand as implausibly strong. They then consider the demand that the semantics should be especially natural or simple, and note, among other things, that it is not out of the question that the only semantics that can be given in one language for another language is quite unnatural and complex. ${ }^{33} \mathrm{~A}$ third possibility they consider is that the semantics offered should in some sense "explain" reference. They consider two versions of this, where the most relevant for present purposes is the version they spend the most time on, according to which the demand is that "the mapping used in the semantics be identical to the unique (privileged) reference relation". They call a semantics satisfying this condition a Reference Semantics. They reject the demand for a Reference Semantics, saying in essence that if the languages at issue employ different concepts of existence then they will relatedly employ different concepts of reference. Let me quote what they say:

The semantic shape of an alien language need not fit easily against the shape of our language. All that matters, for variantists, is that we can understand the coarse-grained truth conditions of their sentences. Their concept of the existence of something does not match ours, and as goes existence so goes reference. It is okay that we cannot provide a semantics for their language in terms of our concept of reference, though of course in terms of their concepts it is trivially true to say 'The word 'table' refers to tables". The basic upshot is that the truth conditions of the sentences of an alien language need not depend on reference in our sense of "reference". ${ }^{34}$

This may seem obviously right. As goes existence, so goes reference - and hence, it may be thought, to the extent that an argument against quantifier variance relies on the demand for a Reference Semantics, it begs the question. However, there are complications. For example, Hirsch and Warren's own characterization of quantifier variance is neutral on the issue of whether some existence concept is privileged. So even if what they argue is correct and there are, in some sense, different reference relations to employ, one could be privileged. But I will focus on other difficulties regarding what Warren and Hirsch say about the Eklund-Hawthorne argument, and I now turn to them.

\footnotetext{
${ }^{33}$ Hirsch and Warren (2019), p. 601.

${ }^{34}$ Hirsch and Warren (2019), p. 604.
} 
A first item of business is to relate this argument back to the collapse argument. Recall Warren's complaint regarding the collapse argument as initially formulated: the sentence

(1) $\exists_{\mathrm{B}} \mathrm{X} \operatorname{chair}(\mathrm{x})$

and the sentence

(3) $\exists$ s $x$ chair(x)

belong to different languages, so how can one and the same argument have (1) as a premise and (3) as a conclusion? But one way of repairing the argument, distinct from the ones discussed above, is to reason first in Biglish, and there conclude

(2) $\operatorname{chair}(\alpha)$,

and then turn to Smallish and there reason that "chair $(\alpha)$ " of Biglish is true, and from there conclude, in Smallish, that there exists something which satisfies "chair(x)" of Biglish. Assuming we can disquote, (3) follows. The question will be whether such moves are justified.

Enter the distinctions drawn earlier, between the strong and the weak theses, and between the views of philosophers $\mathrm{X}, \mathrm{Y}$ and $\mathrm{Z}$. $\mathrm{X}$ is a verbalist and defends only the weak thesis. For $\mathrm{X}$ it will be a kind of empirical claim whether one can take a true putative subject-predicate sentence of the language of another disputant and assume that there is something to which the subject term refers; it will likewise be a kind of empirical claim whether predicates like "chair(x)" plausibly have the same meaning in the languages of the different disputants. Y's and Z's claims, by contrast, concern what possible languages there are, and how these languages compare. In that setting the relevant claim to investigate will concern for example whether a language in which we cannot perform the relevant disquotation will be expressively deficient.

I will not here attempt to assess these different kinds of assumptions. My only point here is that the different claims rely on quite different assumptions, and proper assessment would look very different depending on what claims and assumptions we are focusing on.

\section{Concluding remarks}

Here is what I hope to have accomplished here. I have distinguished and discussed some different theses that go under the name quantifier variance. I have distinguished and discussed some different claims that can be made about significance of quantifier variance. I have discussed some prominent arguments against quantifier variance, the collapse argument and the Eklund-Hawthorne argument, in 
light of these distinctions. One thing I have not done is to try to render categorical judgments about what we should say. I am, as indicated above skeptical of the significance of X's verbalism. That leaves the views of $\mathrm{Y}$ and $\mathrm{Z}$. I used to think that $\mathrm{Y}$ 's position best captured the significance of quantifier variance. But I now incline toward Z's view, and hence towards thinking that it does not really matter whether the languages concerned contain expressions with quantifier meanings.

\section{REFERENCES}

Chalmers, David, David Manley and Ryan Wasserman (eds.): 2009, Metametaphysics, Oxford: Oxford University Press.

Dorr, Cian: 2014, "Quantifier Variance and the Collapse Theorems", The Monist 9: 503-70.

Eklund, Matti: 2008, “The Picture of Reality as an Amorphous Lump”, in Theodore Sider, John Hawthorne and Dean Zimmerman (eds.), Contemporary Debates in Metaphysics, Blackwell, Oxford.

Eklund, Matti: 2009, “Carnap and Ontological Pluralism”, in Chalmers, Manley and Wasserman (2009).

Eklund, Matti: 2014, “On Quantification and Ontology”, Oxford Handbooks Online (2014), www.oxfordhandbooks.com.

Eklund, Matti: 2016, “Carnap's Legacy for the Contemporary Metaontological Debate”, in Stephan Blatti and Sandra Lapointe (eds.), Ontology after Carnap, Oxford University Press, Oxford.

Hale, Bob and Crispin Wright: 2009, "The Metaontology of Abstraction”, in Chalmers, Manley and Wasserman (2009), pp. 178-212.

Harris, J.H.: 1982, "What is so Logical About the "Logical" Axioms?", Studia Logica 41: 159-71.

Hawthorne, John: 2006, "Plenitude, Convention, and Ontology", in Metaphysical Essays, Oxford University Press, Oxford.

Hirsch, Eli: 2002, “Quantifier Variance and Realism”, Philosophical Issues 12: 51-73.

Hirsch, Eli: 2009, “Ontology and Alternative Languages”, in Chalmers, Manley and Wasserman (2009), pp. 231-58.

Hirsch, Eli: 2011, Quantifier Variance and Realism, Oxford University Press, New York.

Hirsch, Eli and Jared Warren: 2019, "Quantifier Variance and the Demand for a Semantics”, Philosophy and Phenomenological Research 98: 592-605.

McDaniel, Kris: 2017, The Fragmentation of Being, Oxford University Press, Oxford.

McSweeney, Michaela: 2017, “An Epistemic Account of Metaphysical Equivalence”, Philosophical Perspectives 30: 270-93.

Sider, Theodore: 2007, "Neo-Fregeanism and Quantifier Variance", Proceedings of the Aristotelian Society, Suppl. Vol. 81: 201-32.

Sider, Theodore: 2011, Writing the Book of the World, Oxford University Press, Oxford.

Turner, Jason: 2010, “Ontological Pluralism”, Journal of Philosophy 107: 5-34. 
Turner, Jason: 2012, “Logic and Ontological Pluralism”, Journal of Philosophical Logic 41: 419-48.

Warren, Jared: 2015, “Quantifier Variance and the Collapse Argument”, Philosophical Quarterly 65: $241-53$ 